Along these lines the supposed antithesis between homopolar and heteropolar reactions disappears. A more detailed discussion of these aspects of the theory of organic reactions will be submitted elsewhere.

Dyson Perrins Laboratory,

R. ROBINSON

South Parks Road, Oxford.

Jan. 18.

¿ J. Chem. Soc., 220 (1941).

z Heller, II. E., Hughes, E. D., and Ingold, C. K., Nature, 168, 909 (1951).

- Dewar, M. J. S., "Electronic Theory of Organic Reactions", 225 (Oxf. Univ. Press, 1949).

- Braude, E. A., Quart. Rev. Chem. Soc., 4, 423 (1950): Nature. 169, 80 (1952).

\section{A New Synthesis of isoFlavones}

EIGHTEEN different isoflavones are known to occur in plants either in the free state or as glycosides; 5 : 7-dihydroxy-4'-methoxyisoflavone (I) (biochaninA) may be taken as a typical example.

Two main methods have been developed for their synthesis. In the first, a deoxybenzoin, for example (II), is converted into a 2-styrylisoflavone, either by direct cinnamoylation ${ }^{1}$ or by acetylation to a 2 methylisoflavone followed by the condensation of the reactive methyl group with benzaldehyde ${ }^{2}$, and, after protecting any free phenolic groups, oxidation by permanganate gives a 2-carboxyisoflavone, which is decarboxylated and the protecting groups removed. The yields at the oxidation stage are poor, and the method is not well suited for the preparation of partially alkylated isoflavones; it has been used for the preparation of genistein, daidzein and $\psi$-baptigenin.

In the second, a deoxybenzoin (II) is directly converted into an isoflavone by treatment with ethyl formate and sodium. In the early experiments ${ }^{3}$, the mixture was heated and the yields were poor; but Venkataraman and others showed ${ }^{4}$ that much better results were obtained at $0^{\circ}$. The chief drawback to this synthesis is that it only proceeds satisfactorily if all phenolic groups in the deoxybenzoin, except that involved in the cyclization, are protected by methylation or benzylation, though it has been claimed $^{5}$ that the deoxybenzoin (II) is directly converted into the isoflavone (I) by this method. We have, however, been unable to prepare $(\mathrm{I})$ in this way, and related reactions involving formylation of deoxybenzoins containing three free phenolic groups have also failed ${ }^{6}$. A modification of this formylation method $^{7}$ using ethyl orthoformate in pyridine proceeds satisfactorily in the cases of a 2-hydroxy-and

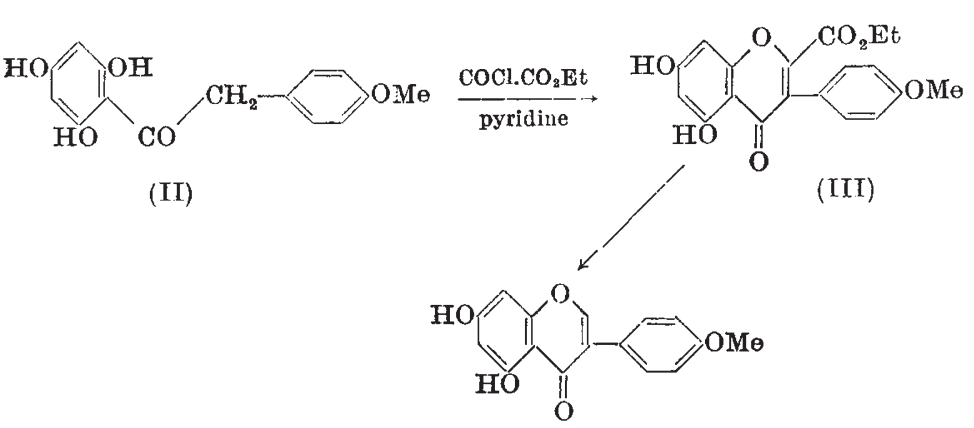

(I) of $2: 4$-dihydroxy-deoxybenzoin, but failed to give an isoflavone with 2:4:6-trihydroxydeoxybenzoin.

We have now developed a new simple isoflavone synthesis starting from a deoxybenzoin (II) which gives good yields at all stages, even when several free phenolic groups are present. It is particularly suited for the preparation of partially alkylated isoflavones without the necessity of employing devices such as partial protection by benzyl groups, partial methylation or partial demethylation. The new method may be illustrated by the preparation of the isoflavone (I). The deoxybenzoin (II), when treated at room temperature with ethyl oxalyl chloride in pyridine, gives directly 2 -carbethoxy-5 : 7-dihydroxy$4^{\prime}$-methoxyisoflavone (III ; melting point, 189 190 ; 60 per cent yield). Mild alkaline hydrolysis then gives the corresponding acid (yield, 98 per cent), which loses carbon dioxide at its melting point, giving the isoflavone (I), best purified as its diacetyl derivative (yield, 81 per cent). Alkaline hydrolysis then gives biochanin-A. The generality of the method has been established by the preparation of a number of other isoflavones, including genistein and $\psi$-baptigenin.

W. BAKER

W. D. OLLIS

(with P. J. L. Binns, J. Chadderton, I. Dunstan, J. B. HARBORNE and D. WeIght).

University, Bristol.

Nov. 23.

${ }^{1}$ Baker, W., and Robinson, K., J. Chom. Soc., 127, 1981 (1925); 2713 (1926); 3115 (1928).

${ }^{2}$ Baker, W., Robinson, R., and Simpson, N. M., J. Chem. Soc., 274 (1933); 805 (1937).

synthesis of $\varphi$-baptigenin, Späth, E., and Lederer, E., Ber., 63, 743

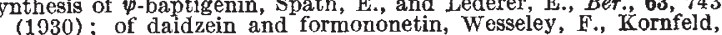
(1930)

4 Venkataraman, K., et al., J. Chem. Soc., 513, 1120, 1769 (1934).

"Shriner, R. L., and Hull, C. J., J. Org. Chem., 10, 228, 288 (1945).

- Venkataraman, K., et al., Current Sci., 18, 404 (1949). Winter, R., dissertation, Bristol (1950). Weight, D. (unpublished observation)

? Venkataraman, K., et al., Current Sci., 18, 373, 404 (1949) (Chem. $A b 8 ., 44.8916,3988$ (1950)).

\section{Separation of the 3,5-Dinitrobenzoates of Volatile Alcohols by Paper Chromatography}

Few methods for the separation of derivatives of volatile alcohols by paper chromatography have been reported. Rice, Keller and Kirchner ${ }^{1}$ have separated a number of 3,5-dinitrobenzoates by using various solvent systems and filter papers. They quote no $R_{F}$ values, however. Kariyone, Hashimoto and Kimura ${ }^{2}$ have separated the potassium xanthogenates of a number of alcohols, using alkaline butanol as solvent.

The following method for the separation of 3,5-dinitrobenzoates has been of use in the detection of volatile aliphatic alcohols dissolved in benzene or petroleum ether, in quantities greater than $10^{-7}$ gm. mole. The nonaqueous two-phase solvent system methanol-heptane was used-methanol (commercial absolute), heptane ('fraction from petroleum'). A method of revealing the derivatives, previously used in column separations ${ }^{3}$, was adapted for the paper technique. Whatman No. 1 paper, soaked in a methanolic solution of Rhodamine $6 G B N .500$ (I.C.I., Ltd.), $20 \mathrm{mgm}$. 